post-operatively. At 3 months, all PA measures except time spent upright $(p=0.009)$ and time spent standing $(p=0.013)$ had recovered. Measures of PA correlated positively with physical and functional domains of HROL, including EORTC-OLO30 Global Health Status, FAACT Trial Outcome Index (TOI) and FACIT-TOI $(p<0.001)$, and inversely with HADS-Depression $(p<0.001)$.

Conclusion There is marked impairment of $\mathrm{PA}$ at the time of hospital discharge and a gradual recovery over 3-6 months. This carries significant implications in a disease where surgical patients may survive $<2$ years. PA measures are suitable outcomes for evaluating the impact of enhanced recovery programmes on functional recovery and HROL.

Competing interests None declared.

\section{PWE-030 IMMUNOCYTOCHEMICAL ASSESSMENT OF INTRA-TUMOUR MICROVESSEL DENSITY IN OESOPHAGOGASTRIC CANCER DOES NOT HAVE PROGNOSTIC SIGNIFICANCE}

doi:10.1136/gutjnl-2012-302514d.30

${ }^{1} \mathrm{R}$ T Gray, ${ }^{*}{ }^{1} \mathrm{M}$ E O'Donnell, ${ }^{2} \mathrm{~J}$ A McGuigan, ${ }^{1} \mathrm{G}$ M Spence. ${ }^{1}$ Upper Gastrointestinal Surgery, The Ulster Hospital, Belfast, UK; ${ }^{2}$ Thoracic Surgery, Royal Victoria Hospital, Belfast, UK

Introduction Intra-tumour microvessel density (IMD), a marker of tumour angiogenesis, correlates with metastasis and poor prognosis in many cancers. In oesophagogastric cancer however, the prognostic significance of IMD assessment remains incompletely investigated. Methods Patients undergoing surgery with curative intent, without pre-operative chemotherapy, were prospectively recruited between February 1999 and August 2000. Immunocytochemical staining of tumour microvessels was undertaken using anti-CD34 (OBEND 10 clone) antibodies. IMD (microvessels per $\mathrm{mm}^{2}$ ) was assessed using a validated "hot-spot" technique. Patients were followed-up over a 10year period using the Northern Ireland Cancer Registry. The relationship between IMD and standard clinicopathological variables was assessed using the Mann-Whitney $U$ test. Univariate survival analysis was calculated using a Cox's proportional hazard model while survival analysis was calculated using Kaplan-Meier estimation and log rank. Results 61 patients were recruited (male $=45$ ) with a median age of 66.0 years (range 39-83). The overall 10-year survival rate was $19.7 \%(n=12)$. IMD was significantly higher in males compared to females (332.93 vs 252.44, $\mathrm{p}=0.04$ ) and adenocarcinomas compared to squamous cell carcinomas (356.10 vs 203.66, $\mathrm{p}<0.001)$. On univariate survival analysis only lymphovascular invasion predicted poor prognosis (HR 2.26, 95\% CI 1.01 to 5.07, $p=0.05$ ). Kaplan-Meier survival analysis demonstrated no difference in longterm survival for patients with IMD levels greater or less than the median value (738 days vs 882 days, $p=0.67$ ).

Conclusion Immunocytochemical analysis of IMD does not have a prognostic benefit in determining long-term survival in patients with oesophagogastric cancer.

Competing interests None declared.

\section{PWE-031 THE POTENTIAL OF HAEM TRANSPORT PROTEINS AS THERAPEUTIC TARGETS IN THE TREATMENT OF OESOPHAGEAL ADENOCARCINOMA}

doi:10.1136/gutjnl-2012-302514d.31

${ }^{1} \mathrm{~S}$ J Ford, ${ }^{* 1} \mathrm{M}$ Bedford, ${ }^{2} \mathrm{O}$ Tucker, ${ }^{3} \mathrm{D}$ Alderson, ${ }^{1} \mathrm{~T}$ H Iqbal, ${ }^{1} \mathrm{C}$ Tselepis. ${ }^{1}$ Cancer Studies, University of Birmingham, Birmingham, UK; ${ }^{2}$ Upper GI Surgery, Birmingham, UK; ${ }^{3}$ Cancer Studies, University Hospital Birmingham, Birmingham, UK

Introduction Epidemiological evidence supports the potential role of dietary haem iron in gastrointestinal carcinogenesis. ${ }^{12}$ Oesophageal cancer cells acquire inorganic and organic iron by progressive upregulation of iron and haem transport proteins. ${ }^{3}$ These alterations result in increased cellular iron loading which is likely to drive cellular proliferation. ${ }^{3}$ The effect of perturbing haem import proteins, hemopexin receptor (LRP1) and haem carrier protein (HCP1), on cancer cell iron transport, phenotype and tumour burden is unknown. The aim was to determine the in vitro and in vivo effects of depriving oesophageal cancer cells of haem iron.

Methods Stable knock-downs of LRP1 and HCP1 were created by infection with specific shRNA lenti-viral vectors. Successful knockdown was confirmed by Western blotting. The effect on cellular iron transport and cell phenotype was assessed by qRT-PCR and phenotypic experiments for viability, proliferation, migration and anchorage independent growth. Stable knock-downs of LRP1 and HCP1 were then xenografted into NOD-SCID mice as an in vivo model of oesophageal adenocarcinoma.

Results Perturbation of LRP1 and HCP1 caused a compensatory upregulation of inorganic iron import proteins and a decrease in iron storage capacity. Neoplastic activity was significantly impaired compared to control (proliferation, viability, colony forming and migration - all $p=<0.01$ ). Loss of active haem iron import significantly reduced xenograft tumour burden in murine models with a $70 \%(p=0.014)$ and $58 \%(p=0.05)$ reduction in average xenograft weight compared to control.

Conclusion Haem import significantly contributes to iron loading in oesophageal cancer cells and creates a more aggressive phenotype. Functional inhibition of haem importing proteins LRP1 and HCP1 curbs neoplastic activity and significantly reduces in vivo tumour burden in murine models. Haem iron transport proteins are potential therapeutic targets in the treatment of oesophageal adenocarcinoma.

Competing interests None declared.

\section{REFERENCES}

1. Cross AJ, Freedman ND, Ren J, et al. Meat consumption and risk of esophageal and gastric cancer in a large prospective study. Am J Gastroenterol 2011:106:432-42.

2. Cross AJ, Leitzmann MF, Gail MH, et al. A prospective study of red and processed meat intake in relation to cancer risk. PLoS Med 2007;4:e325.

3. Boult J, Roberts K, Brookes MJ, et al. Overexpression of cellular iron import proteins is associated with malignant progression of esophageal adenocarcinoma. Clin Cancer Res 2008;14:379-87.

\section{PWE-032 ENDOSCOPIC MUCOSAL RESECTION (EMR) FOLLOWED BY ADJUVANT RADIOFREQUENCY ABLATION (RFA) CAN RESULT IN BETTER OUTCOMES COMPARED TO EMR ALONE IN PATIENTS WITH BARRETT'S EARLY NEOPLASIA (EN). A COMPARATIVE STUDY FROM A TERTIARY CENTRE IN THE UK}

doi:10.1136/gutjnl-2012-302514d.32

${ }^{1} \mathrm{~S}$ Sami, ${ }^{1} \mathrm{E}$ Telakis, ${ }^{1} \mathrm{~J}$ Mannath, ${ }^{2} \mathrm{P}$ Kaye, ${ }^{1} \mathrm{~K}$ Ragunath. ${ }^{1}$ Department of Gastroenterology, Nottingham Digestive Diseases Centre and NIHR Biomedical Research Unit, Nottingham University Hospitals NHS Trust, Nottingham, UK; ${ }^{2}$ Department of Histopathology, Nottingham University Hospitals NHS Trust, Nottingham, UK

Introduction RFA has shown efficacy in eradicating Barrett's EN (high grade dysplasia (HGD) or intra-mucosal cancer (IMC)). To our knowledge, there are no studies directly comparing outcomes in patients with EN who undergo EMR alone vs EMR followed by RFA. The aim of this study was to assess the efficacy, safety and long term outcomes of adjuvant RFA in this setting.

Methods We searched our prospective Barrett's Oesophagus EMR database for patients who had EMR of lesions harbouring EN followed by RFA for eradication of residual Barrett's mucosa between 2007 and 2008 as part of a multi-centre trial (intervention group). The control group included patients with similar lesions 
who had undergone EMR followed by surveillance of residual Barrett's mucosa. The two groups were matched for any potential confounders to minimise bias.

Results There were 13 patients in each group. Mean age in the EMR group and EMR+RFA group was 70 and 59 years, respectively. Both groups were equally matched in terms of male to female ration (12:1); length of circumferential Barrett's mucosa; lesion Paris classification; mean lesion size; and resection type (Piecemeal or Enbloc). The mean duration of follow-up in the EMR group was 21 months compared to 32 months in the EMR+RFA group. The histological characteristics of lesions in both groups are shown in the table below (Abstract PWE-032 table.1). Overall, histological eradication of EN was achieved in eight (62\%) patients in the EMR group and $13(100 \%)$ in the EMR+RFA group at the last follow-up. Persistence or recurrence of EN and the need for further EMR during follow-up occurred in five patients (38\%) in the EMR group (two of them had Oesophagectomy) compared to only one (8\%) in the EMR +RFA group. One patient (8\%) in the EMR group developed oesophageal stricture and no complications occurred in the other group.

\section{Abstract PWE-032 Table 1}

\begin{tabular}{lcc}
\hline Histological characteristics & EMR group $(\mathbf{n}=\mathbf{1 3})$ & $\begin{array}{c}\text { EMR }+ \text { RFA } \\
\text { group }(\mathbf{n}=\mathbf{1 3})\end{array}$ \\
\hline $\begin{array}{l}\text { Pre-EMR lesion histology } \\
\text { HGD }\end{array}$ & $10(77 \%)$ & $10(77 \%)$ \\
IMC & $3(33 \%)$ & $3(33 \%)$ \\
EMR specimen histology & $6(46 \%)$ & $3(33 \%)$ \\
HGD & $7(64 \%)$ & $10(77 \%)$ \\
IMC & $13(100 \%)$ & $13(100 \%)$ \\
Clearance at lesion base & $4(31 \%)$ & $4(31 \%)$ \\
Residual HGD post EMR & $1(8 \%)$ & $1(8 \%)$ \\
Residual LGD post EMR & & \\
\hline
\end{tabular}

Conclusion These data suggest that adjuvant RFA in this setting can have a significant positive impact on the long term success rate of histological eradication of EN in Barrett's Oesophagus as well as reducing the risk of recurrence of those lesions. It can reduce the need for subsequent EMRs and radical surgery with no safety concerns. The long duration of follow-up and control for confounders add significant validity to the results, despite the relatively small number of patients included.

Competing interests S Sami: None declared, E Telakis: None declared, J Mannath: None declared, P Kaye: None declared, K Ragunath Grant/Research Support from: Olympus, Cook and Barrx medical.

\section{PWE-033 COMPREHENSIVE ASSESSMENT OF OUTCOMES INCLUDING COSTS AND SURVIVAL IN YOUNGER VS OLDER PATIENTS UNDERGOING SURGICAL MANAGEMENT OF OESOPHAGEAL CANCER}

doi:10.1136/gutjnl-2012-302514d.33

S R Markar, ${ }^{*}$ A Karthikesalingam, D Low. Esophageal Surgery, Virginia Mason Medical Center, Seattle, Washington, USA

Introduction The aim of this study was to compare disease presentation, clinical and pathological staging, peri-operative outcome, costs and long-term survival of patients 50 years and under $(\leq 50)$, and those over 50 (>50) undergoing oesophagectomy for oesophageal malignancy.

Methods All patients undergoing oesophagectomy by a single surgeon for cancer between 1991 and 2011 had information prospectively entered in an IRB-approved database. These two groups were compared for symptomatic presentation that is, length of dysphagia and degree of weight loss, clinical and pathologic stage, neoadjuvant therapy, medical co-morbidities, operative outcomes including complications, treatment costs and survival.

Results In total 493 patients underwent surgical resection for oesophageal malignancy from 1991 to 2011. 58 of these patients were $\leq 50$ yrs $(44 \pm 4.7)$, and 435 patients were $>50$ years $(67 \pm 8.44)$. Younger patients demonstrated an increased likelihood for delayed presentation as shown by an increased length of dysphagia $(5.79 \pm 13.19$ vs $3.4 \pm 6.97$ months) and increased weight loss $(14.69 \pm 21.12$ vs $10.13 \pm 14.55 \mathrm{lbs})$. Older patients typically presented with more cardiac comorbidities. Clinical stage was similar, the younger cohort of patients demonstrated a significantly increased incidence of adenocarcinoma $(93.1 \%$ vs $82.53 \%)$ and Signet ring pathology (10.34\% vs $6.44 \%$ ). Treatment approach was similar except younger patients were more likely to receive neoadjuvant chemoradiotherapy for stage IIA disease $(53.85 \%$ vs $27.1 \%)$ and chemotherapy alone for stage IIB (42.86\% vs $11.11 \%$ ). Length of operation, blood loss, transfusion requirements and length of hospital stay were similar for both groups. However, patients $\leq 50$ years demonstrated significantly shorter Intensive care unit stay (1.43 \pm 1.08 vs $2.08 \pm 2.59$ days), reduced incidence of postoperative complications (29.31\% vs $48.51 \%$ ) and in subset analysis reduced overall cost ( $\$ 20133 \pm 7048$ vs $\$ 23921 \pm 10787)$. No significant difference was noted in final pathological stage, incidence of complete response to therapy or positive resection margins. Average follow-up was approximately 4 years in the $\leq 50$ age group and 3.5 years in the $>50$ age group with no difference noted in 5 -year survival $(46.15 \%$ vs $38.33 \%(p=0.35)$. Log-rank testing also showed no difference between under 50 and over 50 age groups for all cause mortality during the study period $\left(\chi^{2} 0.432 ; p=0.511\right)$.

Conclusion This study demonstrates younger patients have fewer complications and less overall treatment costs following oesophagectomy. In spite of having a more delayed presentation, and a higher incidence of adenocarcinoma younger patients presented with a similar stage and demonstrated similar overall survival.

Competing interests S Markar Grant/Research Support from: Ryan Hill Research Foundation, A Karthikesalingam: None declared, D Low: None declared.

\section{PWE-034 THE CLINICAL AND ECONOMIC COST OF DELIRIUM FOLLOWING SURGICAL RESECTION FOR OESOPHAGEAL MALIGNANCY}

doi:10.1136/gutjnl-2012-302514d.34

S R Markar,* I Smith, D Low. Virginia Mason Medical Center, Seattle, Washington, USA

Introduction Delirium is an under-estimated and serious complication following major surgery, particularly in the elderly population. The aim of this study was to identify pre-operative risk factors for delirium following oesophagectomy for malignancy, and investigate its impact upon short and long-term outcome.

Methods All patients undergoing oesophagectomy for cancer between 1991 and 2011 had information prospectively entered in an IRB-approved database. Patients were divided into two groups based upon the presence or absence of clinically-significant post-operative delirium, and were compared with respect to use of neoadjuvant therapy, medical co-morbidities, operative outcomes, post-operative complications, overall cost and survival. For the purposes of this study delirium was defined as an acute fluctuating confusional state that required intervention.

Results 500 patients were included in this analysis; 46 (9.2\%) patients with post-operative delirium and 454 patients without. In the delirium group, age was significantly increased $(71 \pm 8.1 \mathrm{yrs}$ vs $63 \pm 10.9 \mathrm{yrs})$ and BMI was reduced $\left(25 \pm 4.2\right.$ vs $\left.27 \pm 4.8 \mathrm{~kg} / \mathrm{m}^{2}\right)$. There were no significant differences in cardiac, pulmonary or renal co-morbidities, however ASA grade (2.8 \pm 0.4 vs $2.6 \pm 0.5)$ and 